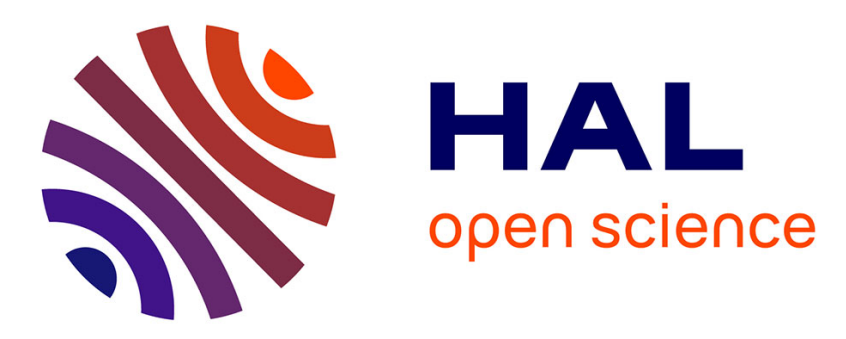

\title{
Software Support Requirements for Awareness in Collaborative Modeling
}

\author{
Michel Dirix, Xavier Le Pallec, Alexis Muller
}

\section{To cite this version:}

Michel Dirix, Xavier Le Pallec, Alexis Muller. Software Support Requirements for Awareness in Collaborative Modeling. On the Move to Meaningful Internet Systems: OTM 2014 Conferences, 2014, Amantea, Italy. pp.382-399, 10.1007/978-3-662-45563-0_22 . hal-01251415

\section{HAL Id: hal-01251415 \\ https://hal.science/hal-01251415}

Submitted on 6 Jan 2016

HAL is a multi-disciplinary open access archive for the deposit and dissemination of scientific research documents, whether they are published or not. The documents may come from teaching and research institutions in France or abroad, or from public or private research centers.
L'archive ouverte pluridisciplinaire HAL, est destinée au dépôt et à la diffusion de documents scientifiques de niveau recherche, publiés ou non, émanant des établissements d'enseignement et de recherche français ou étrangers, des laboratoires publics ou privés. 


\title{
Software Support Requirements for Awareness in Collaborative Modeling
}

\author{
Michel Dirix ${ }^{1,2}$, Xavier Le Pallec ${ }^{1}$, and Alexis Muller ${ }^{2}$ \\ 1 University of Lille1 - France \\ michel.dirix@ed-univ-lille1.fr, \\ xavier.le-pallec@univ-lille1.fr \\ 2 Axellience - France \\ \{michel.dirix, alexis.muller\}@genmymodel.com
}

\begin{abstract}
To address issues of traditional modeling tools (installation, model versioning and lack of model repositories), Axellience has developed the first online UML modeling tool. In GenMyModel's beta-phase, the most requested feature was collaboration. Supporting collaborative modeling involves addressing classical concerns of CSCW. These issues are usually classified through core dimensions like awareness and articulation work. We decided to focus our research on the most important dimension: awareness. Commercial modeling tools and research prototypes provide little support for awareness.To define the importance of awareness in modeling tools, we decided to study what awareness information is really required in collaborative modeling and to assess its importance according to articulation work types. To do this, we have implemented a basic collaboration system without constraint on articulation work. After a few months of use, we have identified three articulation work types present in more than 500 collaborative projects. This preliminary study allowed us to define awareness elements potentially needed for each articulation work type. As these elements are different for each articulation work type, we launched different surveys for each one of them. With these surveys, we have sorted awareness information by relevance according to articulation work types.
\end{abstract}

Keywords: Modeling, Collaboration, Awareness, Survey.

\section{Introduction}

When a person uses a modeling tool, one problem is remembering where models are saved. Furthermore, installing a modeling tool can be time-consuming. When collaborators want to send models between them, they have to ensure that collaborators use the same version of the modeling tool. GenMyModel is an answer to these problems. GenMyMode 1 is an online modeling tool. As it is online, users do not have to install or update the modeling tool. In addition, they do not have to know where their models are saved. While GenMyModel

\footnotetext{
1 http://www . genmymodel.com/ 
was in beta-phase and had only 200 users registered, collaboration was the most requested feature. It was not planned at the beginning of GenMyModel's development to provide collaboration so quickly. We started by adding a basic version of collaboration. As of mid-May, GenMyModel had more than 50,000 users distributed in 200 countries, and more than 5,800 users were involved in more than 3,400 collaboratives projects. Collaboration is widely used and confirms the need of real-time collaboration as requested when GenMyModel had 200 users. Collaboration is above all being aware of others' activities. It implies displaying information about these activities. Thus, supporting collaborative modeling involves addressing classical concerns of CSCW(Computer-Supported Cooperative Work). "CSCW should be conceived as an endeavour to understand the nature and characteristics of cooperative work with the objective of designing adequate computer-based technologies" [1. CSCW contains a number of core dimensions like:

Articulation Work: It consists of articulating distributed work of users. [2].

Awareness: Awareness is one crucial aspect of switching between work in solitude and collaboration. 3. Awareness provides important information about activities of collaborators and interactions with the shared workspace in order to place the user in the best context [4].

Awareness is primordial for collaborative modeling. Nevertheless, awareness is little supported in commercial tools and research work. Therefore, we decided to do a study on this aspect. As awareness is centered on the needs of users, we decided to follow the user-centered design process. Our study is the first step of the process. The goal of our study is to know how modeling tools users work in collaboration. We want to know what the different articulation work types are and what awareness information is needed in these steps. To complete this study, we used GenMyModel and the many collaboratives projects constructed by users and we analyzed them. With this analysis, we determined three articulation work types. Then, for each articulation work type, we surveyed users of modeling tools to evaluate the importance of each awareness information. While we mainly contacted GenMyModel users for the survey, we also included users of other modeling tools in order to generalize our results: we took care to assess whether the modeling tool used influences answers. To go further with our surveys, we researched if correlations between distance between users, the size of the project, the size of the team or the context of the project, and awareness needs exist.

The remainder of the paper is organized as follows. In section 2, we will describe awareness in depth, what are awareness information types and how they are currently handled by commercial modeling tools and research works. In section 3, we will present the study with the analysis of collaborative projects to define different articulation work types. In section 4, we will link articulation work types with awareness information. From these links, we will look at the surveys for modeling tool users in section 5. Finally, we will present the results in section 6 . 


\section{Awareness}

In this section, we will present the CSCW dimension which mainly guides our experiment: awareness. Then, we will see how awareness is supported in modeling tools.

\subsection{Global Definition}

The most widely used definition of awareness is given by Dourish and Bellotti 4 : "[...] an understanding of the activities of others, which provides a context for your own activities". Schlichter 5 built on this aspect of CSCW a few years later in order to reach and to maintain effective coordination of collaborative work. In his review of awareness in Distributed Collaborative Software Engineering [6], Omoronyia defines four types of awareness as follows:

Workspace Awareness. The up-to-minute knowledge of other participants interactions with the shared workspace 7 .

Informal Awareness. The general sense of who is around, what they are doing, and what they are going to do 8 .

Group-Structural Awareness. Knowledge about people roles and responsibilities, their positions on an issue, their status, and group processes 8 .

Social Awareness. Information about the presence and activities of people in a shared environment 9 .

These types refer to a series of questions people ask themselves when they collaborate. Table 1 shows a list of the main ones.

Workspace awareness is the most studied in awareness literature [6], and it seems to also be the most important type in Distributed Collaborative Software Engineering. Even if awareness is the focus of many scientific works for decades, it is still a scientific issue. The April 2013 volume of Computer Supported Cooperative Work [11] presents recent scientific results on awareness in different areas (distributed software development, model versioning, virtual team, social media, etc.). Additionally, the new direction of research relates to recent interactive technologies like large tactile surfaces. Yuill [12] reports case studies about awareness with such technologies.

In this part, we presented awareness information for collaboration. We must now know how awareness is supported in modeling tools.

\subsection{Awareness in Modeling}

In the previous part, we saw that awareness is important for collaboration and awareness involves displaying a lot of information. Now, we would like to know what modeling tools provide collaborative features, how they provide this collaboration and what awareness information they display. Few works exist about awareness in modeling tools. Both industrial tools and research prototypes do not implement collaboration in the same way. On the one hand, users of the 
Table 1. Awareness answers to collaboration raised questions [10]

\begin{tabular}{|l|l|l|}
\hline Awareness dimension & Element & Question \\
\hline \multirow{5}{*}{ Workspace } & Identity & Who is that? \\
\cline { 2 - 3 } & Authorship & Who is doing that? \\
\cline { 2 - 3 } & Action & What are they doing? \\
\cline { 2 - 3 } & Action history & How did that operation happen? \\
\cline { 2 - 3 } & Intention & What goal is that action part of? \\
\cline { 2 - 3 } & Intention history & What goal was that action part of? \\
\cline { 2 - 3 } & Artifact & What object are they working on? \\
\cline { 2 - 3 } & Artifact history & How did this artifact come to be in this state? \\
\cline { 2 - 3 } & Location & Where are they working? \\
\cline { 2 - 3 } & Location history & Where has a person been? \\
\cline { 2 - 3 } & Gaze & Where are they looking? \\
\cline { 2 - 3 } & View & Where can they see? \\
\cline { 2 - 3 } & Reach & Where can they reach? \\
\cline { 2 - 3 } & Event history & When did that event happen? \\
\hline \multirow{5}{*}{ Informal } & Opinion & What is their opinion? \\
\cline { 2 - 3 } & Presence & Is anyone in the workspace? \\
\cline { 2 - 3 } & Presence history & Who was here and when? \\
\hline \multirow{5}{*}{ Social } & Interest level & How interested are they? \\
\cline { 2 - 3 } & Emotional feelings & How are they feeling? \\
\cline { 2 - 3 } & Availability & Are they available? \\
\hline Group-structural & Roles and responsibilities & What are their roles/positions? \\
\hline
\end{tabular}

tool share the same environment and edit the same version of the model. On the other hand, users have to retrieve the version of the model locally, modify it and commit and optionally merge the result if users have modified the same elements. Furthermore, in this study, we did not differentiate modeling tools and diagramming software. The difference between both tool types is that the model made with a modeling tool is valid. With modeling tools, many actions are forbidden for compliance with model specifications contrary to diagramming software. Most desktop modeling tools do not appear in our presentation of modeling tools. They provide a classic revision control system for collaboration. Visio provides the best awareness support, so we decided to present only Visio among the desktop modeling tools.

Commercial Tools. Concerning commercial modeling tools, Gliffy 2 is a webbased tool and provides a desynchronized environment. Each collaborator has a local version of the model. Collaborators do not know if others are editing the model or on what they are working. Awareness information is missing.

Contrary to Gliffy, Visid 3 is a desktop tool but also provides desynchronized collaboration. Until the user saves the model, the user can not see changes done by collaborators. When users are editing the model, they know the number of connected collaborators and their names. If they work on elements close to those

\footnotetext{
2 https://www.gliffy.com

3 http://office.microsoft.com/en-001/visio/
} 
on which their collaborators are working, they are notified that a collaborator has made changes on this element. It involves support of Identity, Presence, Authorship partially and Artifact partially awareness elements. Authorship and Artifact support is partial because if the user is not working on the same diagram as your collaborators, the user can not know who has changed the model.

Like Gliffy, Creately 4 is a web-based tool. Creately provides real-time collaboration like Visio. Available features to display awareness information are the list of connected users with their names, a focus on what elements are selected by collaborators, and comments on model parts. The features concern only real-time information and related awareness elements are Identity, Authorship partially, Artifact partially and Presence. Authorship and Artifact support is partial for the same reason as in Gliffy.

Finally, the tool which has the largest support for awareness is Lucidchart5 which is a web-based tool with real-time collaboration. Lucidchart provides a chat panel where collaborators know who is connected and their names. Users can add comments on model elements in order to assign tasks to themselves or to collaborators. Nevertheless, we do not know if a user is working on a specific task when the user is editing the model. Lucidchart also allows users to revert the model to a revision, but users can not see differences between two versions. Thus, Lucidchart supports many awareness elements: Identity, Authorship, Intention partially, Intention history, Artifact partially, Event History and Presence. Thanks to the revision history, users can know who has changed the model, but they can not see what model elements have changed.

Research Prototypes. The main research works on collaboration in modeling tools are centered on collaboration protocol, model merging, etc. but few deal with awareness and are centered on users. Both of the following research prototypes are web-based and provide real-time modeling.

The first is GEMSjax (a meta-modeling tool) 13. In his works, Farwick briefly discusses awareness. He summarizes it as a chat and events to see which elements are currently selected but without giving more details. GEMSjax supports $A u$ thorship partially and Artifact partially information.

Thum with his work on SLIM [14] is akin to Farwick's work on the point of view of awareness. SLIM is a UML Case Tool for synchronous collaborative modeling based on a lock system to avoid conflicts. When a user clicks on an element, a lock is put in place to prevent modifications by another user. Concerning awareness, SLIM contains a chat to discuss, displays current connected users, and padlocks on model elements locked by other users . With these features, SLIM supports Identity, Presence, Authorship partially and Artifact partially.

Both tools support Authorship and Artifact partially, because if users work on different diagrams, they lose information since they can not see changes.

\footnotetext{
${ }^{4}$ http://creately.com/

${ }^{5}$ https://www. lucidchart.com/
} 
Other works exist on awareness but they deal more with the organization of awareness information. Gallardo [15] provides an ontology to organize workspace awareness information for modeling tools.

Issues. Awareness is a crucial aspect for collaborative work. Nevertheless, tools and research prototypes do not seem to be interested in awareness. A quick look to widespread collaborative authoring tools like Google Drive Applications or Office Web Apps shows that they provide a wider support of awareness: Authorship, Identity, Location, Artifact/Action, Artifact/Action History and Presence. Unfortunately, it seems difficult to assess whether the additional awareness features need to be applied in the modeling context without a thorough investigation.

The problem with tools and research prototypes is the multiplication of widgets in order to display information. However, as display area is limited, it is not possible to have more and more widgets to cover awareness elements. Furthermore, users risk a cognitive overload due to the amount of information. Therefore, we have to know what information is required the most. Then, we have to see if requirements vary not only according to different articulation work types but also according to the size of the team, the size of the project, the type of project which is modeled, the project's context or the distance between collaborators.

To complete these objectives, we have questioned people who have already used collaborative modeling through web surveys. These surveys determined what awareness information is relevant for the user. As we think that the relevance of awareness information varies according to articulation work types, first, we studied what these different articulation work types are. These articulation work types are presented in section 3 . We will see that results on articulation work types allow us to determine what information is relevant for each type in section 4 . In section 5 , we will detail how we have built surveys for each articulation work type. Section 6 will present the results and discussions around them.

In future works, with these requirements, we will be able to implement better software support and also evolve the requirements to improve our knowledge on what is needed for collaborative modeling. We can note that our approach is user-centered with analysis, implementation and tests. This approach seems to be adapted because awareness is mainly a HCI issue.

\section{Defining Articulation Work Types}

The first step of our approach is to define different articulation work types. To do this, we analyzed GenMyModel user projects. In order to analyze GenMyModel user projects, we analyzed the database. We extracted collaborative projects with the following criteria:

- Public, to have the right to analyze the project's content.

- Not cloned from GenMyModel examples to avoid tests projects. GenMyModel examples can be cloned in order to discover GenMyModel's capabilities.

- Recent, in order to contact users about a project they remember. 
From March to mid-May, 508 projects met these criteria. For each project, we built users' activity sessions by analyzing launched actions on their projects as shown in figure 1. With these sessions, we can analyze how users interact with the model, and if they edit the model at the same time. For example, in figure 1. we can see numbers in rectangles. Each rectangle corresponds to a user session where actions were launched, and the number indicates the number of launched actions. We can see that the figure is divided into two parts. In the first, the user is the only one to make changes. In the second, user thampe initialized the project with 9 actions. Then, 2 collaborators joined him on the project, and they started to edit the model at the same time. They stopped for 10 minutes and started again until user angart is alone to modify the model. After angart stopped modifying the model, user thampe took the relay and modified the model by himself.

One contributor session

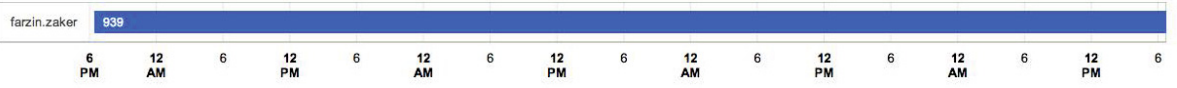

Real-time and turn by turn session

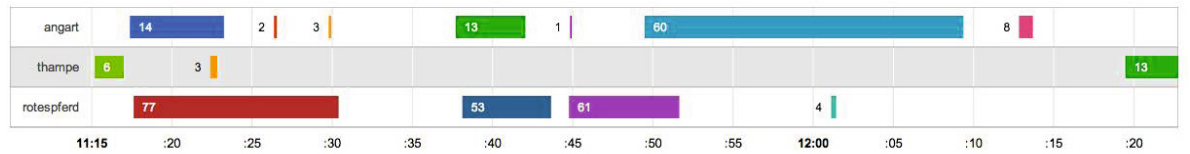

Fig. 1. Examples of sessions

From the project analysis, we determined three articulation work types.

One contributor articulation work (OC): One user modifies the model and the others are spectators. The spectators never interact with the model while the one user is the author of all actions. 308 of the 508 selected projects which satisfied the aforementioned criteria have only one contributor.

Users modify the model turn by turn: (TBT) One user modifies the model and stops modifying. After this, another user does many actions and then stops. The time between a stop and the start of following changes can be one day, a few hours or a few minutes. For example, in a project, we have seen three users take turns every two minutes.

Users modify the model at the same time (RT): More than one user modifies the model in real-time. Real-time collaboration is little used. It represents only $5 \%$ of collaboration time. Nevertheless, this time could be strategic for the coordination between users. We can not know if RT sessions have influenced the following work. Moreover, the utility of RT was not our first objective. 
Often, turn by turn and real-time articulation work types are used in the same project. We defined three different articulation work types, and now we have to link awareness information requirements with them.

\section{Linking Articulation Work Types and Awareness}

In this section, we will discuss what awareness information may be relevant for each articulation work types.

\subsection{One Contributor}

A plausible situation is that the only one contributor is an architect and others are the developers. Developers have to know when parts of the model are finished in order to code them. Furthermore, developers can give their ideas on model improvements. For this articulation work type, the relevant awareness elements are intention and opinion.

\subsection{Users Modify Model Turn by Turn}

Most of the awareness elements are from the past, because no other user has had interaction with the shared environment. The user needs information of what changes took place to understand the actual state of the model. Thus, users may have to know what the collaborators' current tasks are in order not to modify the same model parts. For this articulation work type, the relevant awareness elements are authorship, action history, intention, intention history, artifact history, location history, event history, and presence history. During the analysis of the users activity sessions, we observed another important element. In fact, the time between login and the first command is superior than 1 minute. There are two possible reasons: it is the time needed for users to see all the changes since the last disconnection, or the users look at another browser tab while their model is loading. If the reason is the time needed to see changes since last connection, it means that past events are at least relevant when they log in to their projects.

\subsection{Users Modify the Model at the Same Time}

All awareness information may be needed for real-time collaboration, because the information about curent actions, collaborators, past actions, etc. could be required in order to understand the current state of the model.

Table 2 summarizes the related awareness elements according to the articulation work types. 
Table 2. Possible awareness elements used in articulation work types

\begin{tabular}{|c|c|c|c|}
\hline Element & $\mathrm{OC}$ & TBT & $\overline{\text { RT }}$ \\
\hline Identity & & & $\bar{X}$ \\
\hline Authorship & & $\mathrm{X}$ & $\mathrm{X}$ \\
\hline Action & & & $\mathrm{X}$ \\
\hline Action history & & $\mathrm{X}$ & $\mathrm{X}$ \\
\hline Intention & $\mathrm{X}$ & $\mathrm{X}$ & $\mathrm{X}$ \\
\hline Intention history & & $\mathrm{X}$ & $\mathrm{X}$ \\
\hline Artifact & & & $\mathrm{X}$ \\
\hline Artifact history & & $\mathrm{X}$ & $\mathrm{X}$ \\
\hline Location & & & $\mathrm{X}$ \\
\hline Location history & & $\bar{X}$ & $\mathrm{X}$ \\
\hline Gaze & & & $\mathrm{X}$ \\
\hline View & & & $\mathrm{X}$ \\
\hline Reach & & & $\mathrm{X}$ \\
\hline Event history & & $\mathrm{X}$ & $\mathrm{X}$ \\
\hline Opinion & $\bar{X}$ & $\mathrm{X}$ & $\mathrm{X}$ \\
\hline Presence & & & $\mathrm{X}$ \\
\hline Presence history & & $\mathrm{X}$ & $\mathrm{X}$ \\
\hline Interest level & & & $\mathrm{X}$ \\
\hline Emotional feelings & & & $\mathrm{X}$ \\
\hline Availability & & & $\mathrm{X}$ \\
\hline Roles and responsibilities & & & $\mathrm{X}$ \\
\hline
\end{tabular}

From the table 2, we defined questions to sort awareness elements by relevance for each articulation work type.

\section{Developing Web Surveys}

In the previous section, we defined what awareness elements are concerned according to the articulation work types. Therefore, we discussed with GenMyModel users to define their requirements. To do this, we developed surveys to send to GenMyModel users who had projects which satisfied the aforementioned criteria. Moreover, we shared our surveys on social networks according to the articulation work types. We did this in order to have responses from people who do not use GenMyModel and to know if the use of GenMyModel influences results. Now, we will describe these surveys. For each, we asked them the following information:

- Project context (student project, business project, research project)

- Modeled application type (users model for Desktop, Web or Mobile application)

- Team size (1-3, 3-5, 5-10, 10+)

- How far away are you from your collaborators? (We share the same building, town, country, we are over the world) 
Each question corresponds to a potential element which can be correlated the awareness requirements. We will have to verify further if the correlations found impact the awareness requirements. Now, we will detail the different surveys according to the articulation work type.

\subsection{One Contributor}

Aforementioned possible awareness elements when collaborative projects have just one contributor are intention and opinion. For this articulation work type, we contacted the contributors of 308 projects by email, and we asked the following questions:

- Do you need the opinion of your collaborators?

- If yes, in what context?

- How do you ask their opinion?

- Do others know what your current task is?

- if yes, by what way do they have your current task? How do they use this information?

The first, the second and the third questions determine if opinion is important or not for users and the way to know the opinion of their collaborators. The last questions target intentions. The goal of these questions is not only to know if Opinion and Intention are important for users but also to know how they obtain and share this information.

\subsection{Users Modify Model Turn by Turn and at the Same Time}

As most of the collaborative projects with many contributors use TBT and RT articulation work types, we defined one survey divided into two parts that concerned each articulation work type. In the previous section, we linked the articulation work types with awareness information. Thus, for each awareness information, we had to evaluate the information requirement. We asked the usefulness of awareness information on a scale from 1 to 4 where 1 is useless and 4 is useful. This pair scale forced users to have an opinion on the question. They could not be neutral. To simplify the survey, we generalized awareness information to avoid explaining the sense of each.

For example with RT, for Presence information, we asked the usefulness of Knowing if other collaborators are connected, and for Identity, we asked if the Name of connected collaborators is relevant.

Furthermore, we asked them to give the 5 most important elements aforementioned in order to classify awareness information by relevance. The evaluation of each information on the scale is complementary to the classification, because the evaluation is useful to detect inconsistencies in responses. 
In the second part, with the same type of scale, the respondents evaluated the usefulness of information for TBT. This is when the user works alone on the project. As for RT, we simplified the surveys and instead of asking the usefulness of Presence History, we asked the relevance of Who was connected when you were offline.

Moreover, we asked the question When you open the model, what is the first thing you do? in order to understand why the time between login and the first command is more than one minute. After the questions, we provided a section for comments so that users could offer their ideas about collaboration. For this survey, we contacted 392 collaborators from 508 projects which satisfied the aforementioned criteria. In this email, we asked the person if they wanted to help us to improve collaboration features, and we waited for their responses. It was only to open the discussion. When users were ready to help us, we sent them the online survey made with Google Form. We also posted this form on LinkedIn UML professionals groups in order to have responses from people who do not use GenMyModel. In sharing the survey with others, we wanted to know if the tool influences responses.

\section{Results}

The following results are presented by articulation work types.

\subsection{Ranking Awareness Information by Relevance}

One Contributor. For this first survey, we had 17 responses from the 308 contacted users.
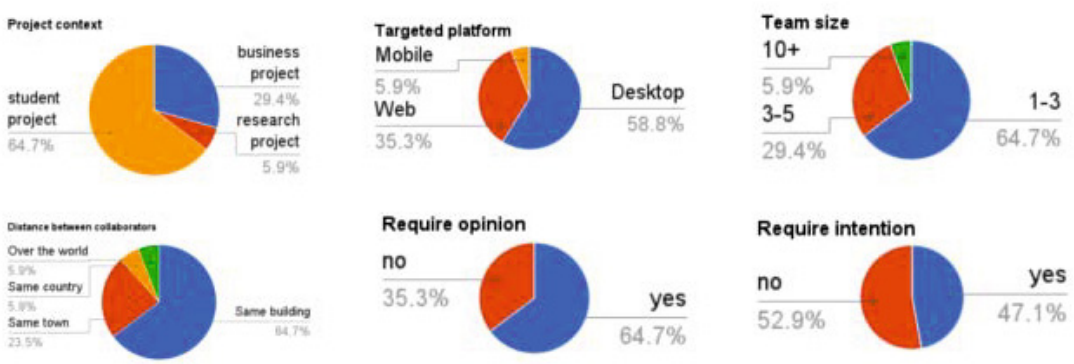

Fig. 2. Survey responses for OC 
Figure 2 summarizes the results of the first survey. We learned that it is mostly students who use the one contributor articulation work type. Therefore, most of the respondents model for desktop applications. Most of the respondents work in little groups, and most of them share the same building. Most respondents need the opinion of their collaborators even if they do not make modifications on the model. For the question, Why do you need the opinion of your collaborators?, they answered that they wanted the advice of others in order to improve the quality of the model.

Concerning intentions, the respondents are more divided. Less than half shared their tasks with others. For these users, all tasks were written in a product and issue tracking product, in a Facebook group or were defined during meetings.

Users Modify Model Turn by Turn. With this survey, we had 50 responses, 35 are GenMyModel users contacted by email, and 15 others came from LinkedIn professional groups for modeling. As figure 3 shows, the three most important awareness elements when users modify the model turn by turn are Action History/Artifact History, Authorship and Intention.

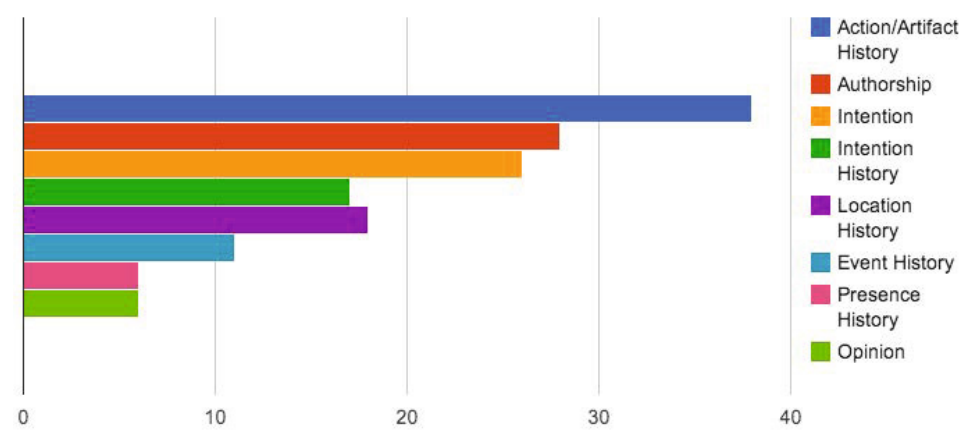

Fig. 3. Ranking of responses for TBT

Users Modify the Model at the Same Time. Figure 4 shows the ranking of each element defined as the most important by users. The most required awareness information for RT is Presence, Identity, Action/Actifact, Intention and Authorship.

Discussions. If we compare the required awareness elements and the existing information in modeling tools, we can see there is a gap. For OC, even if the number of respondents is low, responses give some cues about how they collaborate with only one contributor. On the one hand, modeling tools have to provide support with issue tracking tools like Jira, Mantisse or their own solution, because half of the users share their tasks with their collaborators. On the other 


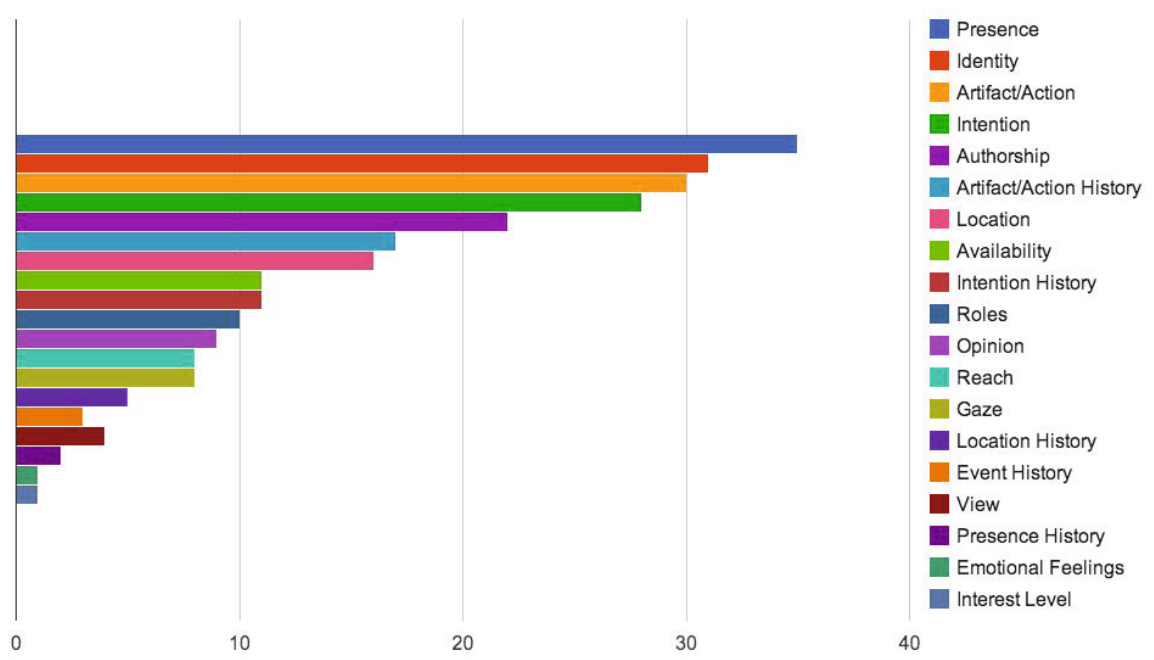

Fig. 4. Ranking of responses for real-time collaboration

hand, as opinion is important, modeling tools have to find a way to retrieve the opinion of collaborators. Currently, the modeling tool with the largest support of awareness is Lucid Chart which provides task features. Nevertheless, Lucid Chart does not allow users to obtain the opinion of collaborators. It is also interesting to mention that tasks and opinions are missing in collaborative authoring tools.

For TBT, the most important awareness information is Artifact/Action History. This information is not covered in modeling tools and research prototypes. As users work alone in TBT, they do not need information about the current changes or their collaborators. Thus, it is logical to have information concerning Artifact/Action History first. The second most important information is related to the first. Respondents want to know the past changes, and furthermore, they want to know who has made these changes. Finally, users want to know what is the current tasks of their collaborators. It can be easily explained. Users do not want to modify model parts which can be modified by others. This information is the only one which is provided by Lucid Chart, but only partially, because it is not possible to know if users are working on tasks. We can see that modeling tools provide a little support for TBT. Even research works in collaborative modeling do not cover the most important awareness information for TBT. In general, the ontology of Gallardo does not refer to past events. Concerning collaborative authoring tools, their awareness support seems fit to TBT as the two most requested information are provided. However, tasks are still missing.

Concerning RT, the most important awareness elements are Presence, Identity, Action/Artifact, Intention and Authorship. As changes are not related to tasks, and users can not follow changes on another diagram, Lucid Chart does 
not cover Action and covers partially Intention and Authorship. As for TBT, collaborative authoring tools do not provide information about tasks.

At the beginning of our study, we observed that the time between login and the first modification is more than one minute. The first thing users do when they log in to the project is to look at the last changes since their last disconnection. Nevertheless, the past awareness information does not appear in the most required information defined by users. It means that they need this information only when they log in to be aware of the project events since their last disconnection.

In section 2, we said that workspace awareness is the most studied in awareness literature. The importance of workspace awareness is confirmed for modeling tools with our study. Most of the important awareness information in the ranking is workspace awareness information.

\subsection{Correlations with the Awareness Requirements}

For each articulation work type, we researched if the size of the team, the size of the project, the modeled application type, the context of the project, the distance between collaborators or if being a GenMyModel user (named elements in the rest of the article) were correlated with awareness information requirements. To do this, we tested the correlation with responses where respondents had to answer on a scale from 1 to 4 . To complete the correlation test, we used a Pearson Correlation test with the SPSSStatistics tool. This test gives correlations between 2 variables. To begin with the tests, we verified that elements were independents. As elements were independents, we verified if they change awareness requirements for each articulation work type.

One Contributor. The Pearson Correlation tests did not reveal links with intention or opinion awareness information. As the number of respondents is low, it is not surprising. It could be interesting to go further with this study.

Users Modify Model Turn by Turn. We present the correlations with awareness requirements for TBT. All of them are resumed in figure 5. In this figure, solid arrows show that the awareness information requirement increases with the element. The values inside brackets mean that the requirement increases with the conditions represented by these values. Significance levels are indicated with stars $(* * *$ for $0.01, * *$ for 0.05 and $*$ for 0.10$)$.

For example, in the figure, we can see that opinion requirement increases with the size of the team. We can also see that Presence History information is more important for users who model a desktop application. 


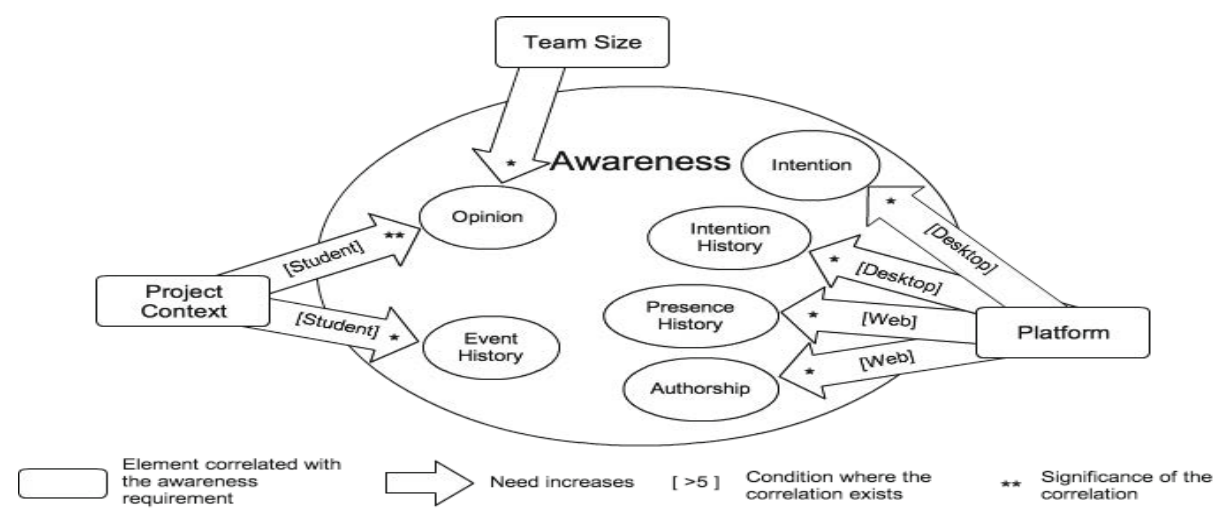

Fig. 5. Correlation between parameters and awareness elements when TBT

Users Modify the Model at the Same Time. We present the correlations with awareness information requirements for RT. Figure 6 shows these correlations. In this figure, dotted arrows mean that the awareness information requirement decreases with the associated element.

Discussions. For TBT, the opinion requirement is higher when the size of the team increases. We are not able to explain why and we will have to discuss this with modeling tool users to understand. In the case of a student project, requirements for Opinion and Event History are more important. It is not surprising, because students are not modeling experts. They need more information from others to build their models together. If modeling tools asked at the creation of the project what the type of project it is, it could be possible to customize displayed awareness information. With this option, users would only see the information important to their projects without useless information. Furthermore, for Intention History information which is at the fifth position in the ranking, the need increases when users model for a desktop application. Currently, we do not have an explanation about the correlation between modeled application type and awareness information. We have to examine user behavior in depth to understand this correlation. Nevertheless, in the case of users who model a desktop application, the ranking could be a little different.

For RT, the size of the team and the size of the project are widely correlated with the awareness information requirements. When the size of the project increases, users have more of a need to know intentions and changes done on the model. It is not surprising, because they have to organize themselves for a large project and know the last changes to understand the current state of the model. As intention and past changes on the model are already well ranked, it is very important to highlight this information when the project is large. The larger the project becomes, the more important this information is. As for TBT, opinion requirement increases with the size of the team. 


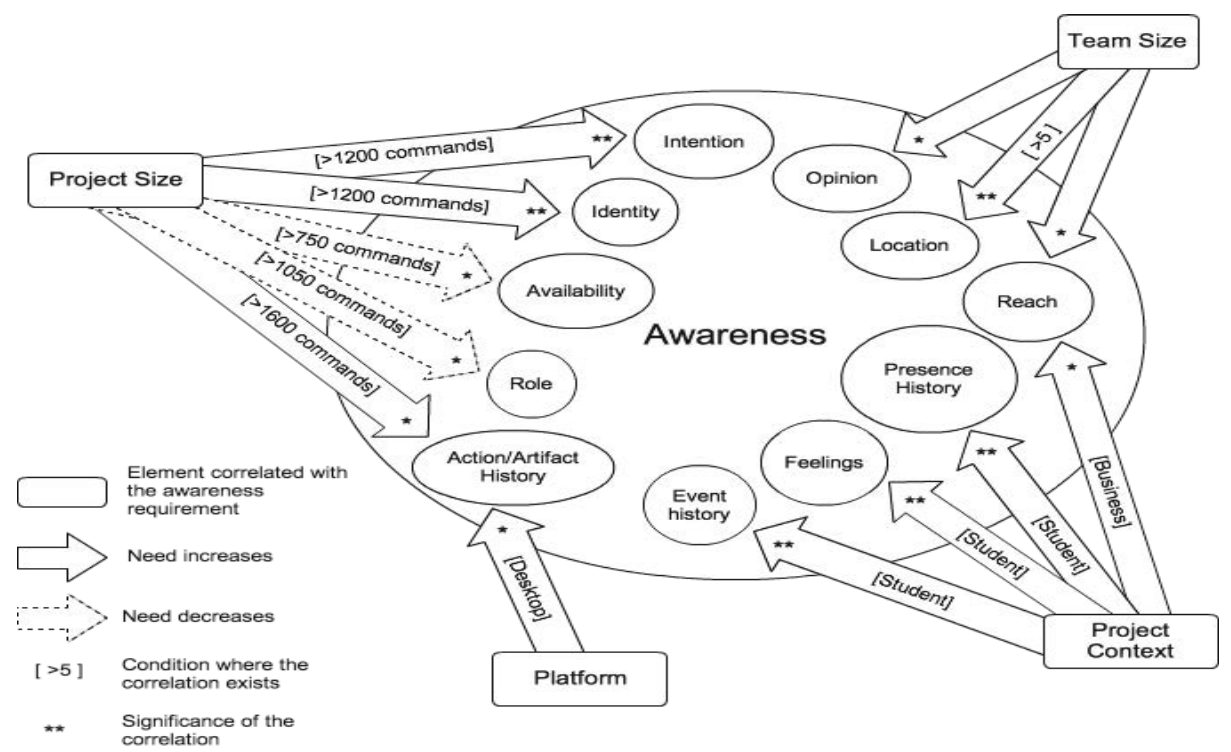

Fig. 6. Correlation between parameters and awareness elements for RT

As the tool used by the survey respondents is not correlated with the results, the rankings and the correlations are applicable for all modeling tools.

Despite correlations exist with the awareness information requirement, modeling tools and research work do not vary the display of awareness information. The same observation is valid for collaborative authoring tools. We will have to realize other studies with modeling tool users to understand why the modeled application type is correlated with awareness information requirements. With these studies, we could refine ranking according to the aforementioned correlations.

Furthermore, in this study, we did not test the correlations of multiple elements at the same time, it will be the topic of another study.

\section{Conclusion}

In this paper, we have seen that GenMyModel was developed to counteract installation problems which can be time-consuming, and the fact that users have to ensure they use the same version of the modeling tool to share their models. As CSCW is user-centered, particularly awareness, we focused our research on this dimension. We looked at awareness support in commercial modeling tools and research prototypes. They provided a little support of awareness. We decided to launch a study with modeling tool users by following the user-centred design process. To begin the study, we analyzed user projects built with GenMyModel in order to categorize how users collaborate, and we defined three articulation work 
types: projects with only one contributor and projects with many contributors, divided into two categories. On the one hand, we have projects which use a real-time collaboration feature, on the other hand, projects where users edit the model turn by turn. For each articulation work type, we defined what awareness elements could be needed for users.

After this analysis, we have the following contributions. The first is the ranking of awareness elements according to the articulation work type. The ranking is different for each. First, for projects which have only one contributor, the awareness elements required are intention and opinion. Then, for the turn by turn articulation work type, the most important awareness information is artifact/action history, authorship and intention. To finish, the real-time articulation work type requires the support of presence, identity, artifact/action, intention and authorship.

The second contribution is the correlations between elements and the awareness requirements. In fact, for RT, the size of the project, the size of the team, the modeled application type and the context of the project increase/decrease the Intention, Identity, Availability, Role, Artifact/Action History, Opinion, Location, Reach, Presence History, Feelings and Event History requirements. For TBT, the requirements are correlated with the size of the team, the context of the project and the modeled application type. The correlated requirements are Opinion, Event History, Intention, Intention History, Presence History and Authorship. We will have to verify these correlations when we implement the awareness information. We can then verify if the awareness requirements change according to aforementioned elements. Except Opinion and Intention, all required awareness features for collaborative modeling have already been proposed by collaborative authoring tools. It would be interesting to study to what extent these features may catch the interest of users of these tools.

As it is not possible to display too much information due to a limited display space, and in order to prevent the cognitive overload, we will study if navigation is possible between the most important awareness elements. For example, when the collaborators' identities are displayed, what possible awareness elements could be displayed next to the users?

First, we will have to define a navigation system for awareness information, and then, we will find out how to display this information. To do this, we will follow the next two steps of the user-centered design process, implementation and tests.

\section{References}

1. Bannon, L.J., Schmidt, K.: Studies in computer supported cooperative work, pp. 3-16. North-Holland Publishing Co., Amsterdam (1991)

2. Schmidt, K., Bannon, L.: Taking cscw seriously. Computer Supported Cooperative Work (CSCW) 1(1-2), 7-40 (1992)

3. Herrmann, T., Nolte, A., Prilla, M.: Awareness support for combining individual and collaborative process design in co-located meetings. Computer Supported Cooperative Work (CSCW) 22(2-3), 241-270 (2013) 
4. Dourish, P., Bellotti, V.: Awareness and coordination in shared workspaces. In: Proceedings of the 1992 ACM Conference on Computer-Supported Cooperative Work, CSCW 1992, pp. 107-114. ACM, New York (1992)

5. Schlichter, J., Koch, M., Xu, C.: Awareness - the common link between groupware and community support systems. In: Ishida, T. (ed.) Community Computing and Support Systems. LNCS, vol. 1519, pp. 77-93. Springer, Heidelberg (1998)

6. Omoronyia, I., Ferguson, J., Roper, M., Wood, M.: A review of awareness in distributed collaborative software engineering. Software: Practice and Experience 40(12), 1107-1133 (2010)

7. Gutwin, C., Stark, G., Greenberg, S.: Support for workspace awareness in educational groupware. In: The First International Conference on Computer Support for Collaborative Learning, CSCL 1995, pp. 147-156. L. Erlbaum Associates Inc., Hillsdale (1995)

8. Gutwin, C., Greenberg, S., Roseman, M.: Workspace awareness in real-time distributed groupware: Framework, widgets, and evaluation. In: Proceedings of HCI on People and Computers XI, HCI 1996, pp. 281-298. Springer, London (1996)

9. Prinz, W.: Nessie: An awareness environment for cooperative settings. In: Bødker, S., Kyng, M., Schmidt, K. (eds.) ECSCW 1999, pp. 391-410. Springer Netherlands (1999)

10. Steinmacher, I., Chaves, A., Gerosa, M.: Awareness support in distributed software development: A systematic review and mapping of the literature. Computer Supported Cooperative Work (CSCW) 22(2-3), 113-158 (2013)

11. Kolfschoten, G., Herrmann, T., Lukosch, S.: Differentiated awareness-support in computer supported collaborative work. Computer Supported Cooperative Work (CSCW) 22(2-3), 107-112 (2013)

12. Yuill, N., Rogers, Y.: Mechanisms for collaboration: A design and evaluation framework for multi-user interfaces. ACM Trans. Comput. 19(1), 1:1-1:25 (2012)

13. Farwick, M., Agreiter, B., White, J., Forster, S., Lanzanasto, N., Breu, R.: A webbased collaborative metamodeling environment with secure remote model access. In: Benatallah, B., Casati, F., Kappel, G., Rossi, G. (eds.) ICWE 2010. LNCS, vol. 6189, pp. 278-291. Springer, Heidelberg (2010)

14. Thum, C., Schwind, M., Schader, M.: Slim-a lightweight environment for synchronous collaborative modeling. In: Schür, A., Selic, B. (eds.) MODELS 2009. LNCS, vol. 5795, pp. 137-151. Springer, Heidelberg (2009)

15. Gallardo, J., Molina, A.I., Bravo, C., Redondo, M.A., Collazos, C.A.: An ontological conceptualization approach for awareness in domain-independent collaborative modeling systems: Application to a model-driven development method. Expert Systems with Applications 38(2), 1099-1118 (2011); Intelligent Collaboration and Design. 